\title{
YKL-40 IS A CSF BIOMARKER OF INTRATHECAL INFLAMMATION IN SECONDARY
}

\section{PROGRESSIVE MS}

\section{Short title}

YKL-40 is a CSF biomarker of SPMS

\section{Authors and affiliations}

Joachim Burman ${ }^{1,2}$, Raili Raininko³, Henrik Zetterberg4,5, Markus Axelsson6, Claes Malmeström6.

${ }^{1}$ Department of Neurosciences, Uppsala University, Uppsala, Sweden.

${ }^{2}$ Department of Neurology, Uppsala University Hospital, Uppsala, Sweden.

${ }^{3}$ Department of Radiology, Uppsala University, Uppsala, Sweden.

${ }^{4}$ Department of Psychiatry and Neurochemistry, the Sahlgrenska Academy at the University of Gothenburg, Mölndal, Sweden.

${ }^{5}$ UCL Institute of Neurology, Queen Square, London WC1N 3BG, United Kingdom.

${ }^{6}$ Department of Clinical Neuroscience and Rehabilitation, University of Gothenburg, Gothenburg, Sweden

\section{Corresponding author}

Joachim Burman, MD, PhD

Address Uppsala University Hospital, SE-751 85 Uppsala, Sweden

Phone $+46(0) 1861115025$

Fax $+46(0) 186115027$

Email: joachim.burman@neuro.uu.se

Word count: 3089 


\section{Abstract}

\section{Background}

Biomarkers of disease activity in secondary progressive MS are scarce. YKL-40 is a glycoprotein predominantly produced by reactive astrocytes in chronic active lesions and is readily detected in cerebrospinal fluid. Thus YKL-40 could be a good biomarker to monitor disease activity in secondary progressive MS.

\section{Methods}

YKL-40 levels in cerebrospinal fluid samples from two independent patient cohorts of MS patients were determined together with two other potential biomarkers of astrocyte and microglia activation (GFAp and sCD14). Patients from one of the cohorts underwent MRI investigation as well.

\section{Results}

YKL-40 levels were overall higher in MS patients than in controls, particularly in secondary progressive MS patients and patients with relapse, whereas relapsing-remitting MS patients in remission had levels indistinguishable from controls. The number of T1, T2 and gadolinium enhancing lesions correlated with the levels of YKL-40, whereas the signal intensity of normal appearing white matter was inversely related to YKL-40. No statistically significant differences between the levels of GFAp and sCD14 in MS patients versus controls were detected, but these correlated moderately with YKL-40, and GFAp correlated strongly with measures of atrophy.

\section{Conclusions}

YKL-40 levels in CSF are increased in secondary progressive MS. YKL-40 is a promising biomarker for therapeutic effect of drugs targeting CNS inflammation in secondary progressive MS.

Keywords: multiple sclerosis, magnetic resonance imaging, 


\section{Introduction}

Multiple sclerosis (MS) is an autoimmune disease of the central nervous system. At onset, $85 \%$ of patients will have a relapsing remitting form (RRMS), with periods of worsening followed by periods of recovery and stable disease. Eventually, most patients will develop a secondary progressive form (SPMS), characterized by fewer and milder relapses $(1,2)$, less radiological activity $(3,4)$, but continuous deterioration of neurologic function (5). Treatments targeting adaptive immunity have largely been unsuccessful in SPMS (6) and at present there is no effective therapy for SPMS.

The cause of SPMS is unknown. The copious treatment failures with anti-inflammatory drugs tell us that if inflammation is still important to the pathogenesis of SPMS, it must be of a fundamentally different form. Chronically active white matter lesions are thought to be characteristic of progressive forms of MS. They contain a demyelinated core with few T cells, which is surrounded by a rim of microglia nodules and enlarged astrocytes $(7,8)$. This type of lesion is also called slowly expanding lesion and is believed to increase in size over time, contributing to demyelination and axonal damage.

YKL-40 (also known as chitinase 3-like-1 or CHI3L1) is a member of the chitinase-like glycoprotein family and is predominantly produced by reactive astrocytes $(9,10)$. In brain tissue from MS patients, YKL-40 is expressed in reactive astrocytes and microglia at the rim of chronically active lesions (10). The physiological role of YKL-40 is not known, but it has been hypothesized to be involved in tissue remodeling during inflammation (9). Increased levels have been found in CSF from patients with clinically isolated syndrome (CIS) and MS (9-15).

The purpose of this study was to further characterize YKL-40 in different stages of MS; to investigate the relation between YKL-40 and other biomarkers of astrocyte and microglia activation; and to explore its potential as a biomarker of inflammation in SPMS.

\section{Materials and methods}

\section{Ethics statement}

The study was approved by the ethics committees of Uppsala University (DNr 2008/182) and Gothenburg University (EPN S589-03, EPN S464-97). All subjects provided written informed consent.

\section{Subjects}


Subjects were prospectively recruited from two university hospitals in Sweden. Cohort A (n=62) was recruited at Uppsala University Hospital. Cohort B $(n=65)$ comprised patients from Sahlgrenska University Hospital, Gothenburg, and has been described previously (13).

\section{Cohort A}

Sixty-two MS patients were included in cohort A: 27 RRMS patients in remission, 15 RRMS patients with a clinical relapse and 20 with SPMS. All patients met the revised McDonald's criteria for MS diagnosis (16). A clinical relapse was defined as new or recurrent neurologic symptoms lasting $\geq 24$ hours, not associated with fever or infection. Relapses were assessed by an experienced neurologist (JB), who also performed all expanded disability status scale (EDSS) scoring (17). SPMS patients were clinically deteriorating in the absence of clinical relapses. No transitional cases of SPMS were included. Five patients with a clinical relapse were investigated twice, in remission and in relapse. The characteristics of subjects are summarized in Table 1.

To identify inflammatory disease activity, MRI of the brain was performed. All but six patients underwent MRI of the entire spinal cord as well. A lumbar puncture was made within one week of the MRI. Following lumbar puncture, CSF was stored in polypropylene tubes stored at $-80^{\circ} \mathrm{C}$ without being thawed and re-frozen. Twenty RRMS patients were on treatment (glatiramer acetate, $n=8$; beta interferon, $n=6$; IVIG, $n=4$; natalizumab, $n=2$ ) and two SPMS patients (interferon beta, $n=1$; natalizumab, $n=1$ ). No patient was treated with corticosteroids within one month of sampling.

\section{Cohort B}

Cohort B consisted of 11 RRMS patients in remission, 29 RRMS patients with a clinical relapse, and 15 SPMS patients CSF was collected by lumbar puncture before immunomodulatory treatment. MRI data were not available from patients in cohort B.

\section{Controls}

Thirty healthy volunteers were recruited as controls. They underwent only lumbar puncture.

\section{Enzyme-linked immunosorbent assays}

All samples were analyzed in the same laboratory by board-certified laboratory technicians who were blinded to clinical data. CSF levels of YKL-40 and sCD14 were analyzed with commercial ELISAs (R\&D systems, Minneapolis, MN). The intra-assay CV was $4.6 \%$ and the inter-assay CV was $6.0 \%$ for YKL-40. The limit of detection was $3.55 \mathrm{pg} / \mathrm{ml}$. GFAp was analyzed with a previously described ELISA (18). 


\section{Acquisition of images}

All MRI investigations were performed with the same imager operating at $1.5 \mathrm{~T}$ using the same imaging protocol. Gadopentetate dimeglumine (Magnevist®, $0.4 \mathrm{ml} / \mathrm{kg}$ body weight, $i$ e double dose) was used as a contrast agent. Technical details regarding the MRI investigations are found in the supplementary section.

\section{Analysis of images}

Technical details regarding the MRI investigations are available in the Appendix. An experienced neurologist (JB) and a senior neuroradiologist (RR) analysed all MR images visually. T2 lesions $>3 \mathrm{~mm}$ in diameter were counted in fluid attenuated inversion recovery images using T2-weighted and proton density-weighted spin echo images as aid. The width of the third ventricle was measured in coronal projections of the cerebrum. The sizes of the lateral ventricles and peripheral cerebral CSF spaces on each side were graded from one to five according to a method described previously (19). In order to assess tissue damage not visible to the naked eye, signal intensities in normal appearing white matter (NAWM) were measured by the neuroradiologist in T2-weighted SE images. Measurements were made in one slice traversing the cerebrum and in another slice traversing the cerebellum and pons. In the cerebrum, the regions of interest were placed in the frontal lobe anterior to the frontal horn, in deep white matter near the atrium of the lateral ventricle, and in the base of a lower parietal gyrus. In the other slice, the ROIs were placed in the cerebellum in close proximity to the middle cerebellar peduncle and in the anterior pons. The intention was to normalize the measured SIs of ROIs to the SIs of the references in the same slice. However, the references were not always optimally placed and could not be used in all slices selected for measurements. Assessment of NAWM with this method could be made in 37/64 examinations using the oil reference and 42/64 examinations using the water reference.

\section{Statistical analysis}

Statistical analyses were done with GraphPad Prism 6.0 (GraphPad Software, La Jolla, CA) and SAS 9.3 (SAS Institute, Cary, North Carolina, USA). Baseline characteristics were summarized by MS subtype using frequencies for categorical variables and medians with interquartile intervals for continuous variables. To determine statistically significant differences between two groups, the Mann-Whitney test was used; for statistical significance between three or more groups the Kruskal-Wallis test. Dunn's multiple comparison test was used for post hoc analysis. Correlations were described with Spearman's rank correlation coefficient. An ANCOVA model with the natural logarithm of YKL-40 as dependent variable and MS subtype and age as independent variables was used. Pairwise comparisons were performed using Tukey's method. Geometric means were calculated using 
the antilogs of the model-adjusted means of the log transformed YKL-40 levels. A two-tailed $p$ value of $<0.05$ was considered significant. All described differences are statistically significant unless otherwise stated.

\section{Results}

\section{Results from analyses of Cohort A}

\section{MRI findings}

All patients with a clinical relapse had a least one gadolinium enhancing $(\mathrm{Gd}+)$ lesion on T1 sequences. In addition nine RRMS patient in remission had subclinical inflammatory activity with at least one Gd+ lesion. These patients were grouped together in the subsequent analyses. The results of the analysis of the MRI measurements are summarized in Supplementary table 3 of the Appendix.

\section{$Y K L-40$ in relation to clinical data}

SPMS patients as well as RRMS patients with Gd+ lesions had increased levels of YKL-40 in comparison to RRMS patients in remission and controls (Fig 1A). Patients in relapse had higher levels than when the same patients were examined in remission (Fig 1B). SPMS patients with $\mathrm{Gd}+$ lesions $(\mathrm{n}=3)$ had higher values than other SPMS patients (mean $257 \mathrm{vs} 146 \mathrm{ng} / \mathrm{mL}$ ) but due to the small number of patients in this group, no statistical analysis was made. YKL-40 correlated with EDSS scores (Spearman $\mathrm{r}=0.32$, $\mathrm{p}=0.0089$, but not with disease duration. A strong correlation with age was found when the controls were examined separately (Spearman $r=0.68, p<$ 0.0001). Therefore, an age-adjusted analysis was made. After correction for age, YKL-40 values from the SPMS group were no longer different with statistical significance from RRMS patients in remission.

\section{YKL-40, GFAp and $S C D 14$ in relation to MRI data}

In order to determine the relationship between YKL-40, GFAp and SCD14 vis-a-vis ongoing inflammation, manifest tissue damage and tissue damage in NAWM a set of correlation analyses was made (Table 2). YKL-40, but not GFAp and sCD14, was correlated with the number of Gd+ lesions and accordingly YKL-40 levels were higher in patients with more Gd+ lesions (Figure 1C). For the subsequent analyses, patients with Gd+ lesions were excluded in order to remove the influence of acute inflammation. YKL-40 correlated with the number of T1 lesions, T2 lesions and atrophy of cerebral sulci. In addition, YKL-40 was negatively correlated with the SI of NAWM. GFAp correlated with the number of T1 and T2 lesions as well as all measures of atrophy. No significant correlation was seen between the levels of sCD14 and any MRI parameter. 


\section{Results from the combined cohorts}

Results of analyses of YKL-40 and sCD14 in cohort B have been reported previously (13). The levels of YKL-40 were higher in MS patients from the combined cohorts $(A+B)$ than in controls (median 121 vs 82.0, $p<0.0001$ ). For the subsequent analyses, RRMS patients with presence of $\mathrm{Gd}+$ lesions from Cohort A were analyzed together with patients with clinical relapse from Cohort B and patients with RRMS and no Gd+ lesions from Cohort A were analyzed together with RRMS patients in remission from Cohort B. After an age-adjusted analysis, SPMS patients had higher YKL-40 levels than controls. In addition, patients with Gd+ lesions and clinical relapse had increased levels of YKL-40 in comparison with RRMS patients in remission and controls (Figure 1D). The levels of YKL-40, GFAp and sCD14 correlated with each other to a similar degree (Table 3). GFAp in MS patients was not different from controls ( 321 vs $290 \mathrm{pg} / \mathrm{mL}, \mathrm{p}=0.34$ ); nor was sCD14 (84,7 vs $98,5 \mathrm{ng} / \mathrm{mL}, \mathrm{p}=0.11$ ).

\section{Discussion}

We have previously reported that YKL-40 is increased in the CSF from MS patients (13). Our main finding in this study is that YKL-40 is elevated in CSF from SPMS patients, similar to the level of RRMS patients with relapse. This finding supports that astrocyte-microglia interactions are important to the pathogenesis of SPMS and that inflammation remains important in this chronic phase of the disease. Since other biomarkers of inflammation such as presence of $\mathrm{Gd}+$ lesions or intrathecal production of chemokines are generally absent or low-grade in SPMS $(3,4,20)$, this is consistent with the idea of compartmentalized inflammation.

For the purposes of this study we propose a model where the mechanism of increased YKL-40 production is fundamentally different in the different stages of MS. In the early stages of RRMS, increase in YKL-40 production would be dependent on the presence of acute lesions, which can be determined in vivo by MRI investigation with administration of a contrast agent such as gadolinium. In this setting, YKL-40 production should be low in patients while in biological remission (i.e. no clinical relapse and absence of $\mathrm{Gd}+$ lesions at MRI investigation) and high in patients with a biological relapse. With the accumulation of chronically active lesions, leading to the phenotype of SPMS, the baseline level of YKL-40 should increase, reflecting low-grade inflammation in these lesions, ultimately leading to tissue damage and disability.

This model is supported by data from several studies. In vivo, YKL-40 is found in the soma of astrocytes in the vicinity of microglial nodules (21). It has been demonstrated that YKL-40 mRNA is expressed by reactive astrocytes surrounding microglial nodules suggesting that macrophages induce YKL-40 expression in 
surrounding astrocytes (9). In the Gd+ lesion massive infiltration of macrophages/microglia is seen together with reactive astrocytes, whereas in chronic inactive lesions microglia are sparse (22). Further, microglial nodules are found in white matter bordering chronically active lesions from SPMS patients where ongoing demyelination is present $(7,8)$ - when absent, demyelination and axonal degeneration are similar to agematched controls (8). In vivo, SPMS patients have on average more activated microglia in T1 lesions than RRMS patients (23). Lastly, increased levels of YKL-40 in the CSF from MS patients confer an increased risk of progression to EDSS 3 and 6 (14).

Our data are supportive of this model. We have previously shown that YKL-40 is increased in all MS patients, regardless of subtype (13). In this study, with the addition of MRI data, we were able to further refine this analysis. RRMS patients in remission without Gd+ lesions had YKL-40 levels indistinguishable from controls. Notably, three outliers had clearly elevated levels, which may represent subclinical relapses with CNS inflammation that were not detected by MRI or incipient SPMS (Figure 1A). Patients in clinical relapse and/or with Gd+ lesions displayed increased levels of YKL-40. The dynamic fluctuation of YKL-40 in RRMS is further reinforced by the finding that YKL-40 levels decreased with repeated investigation when patients were investigated in remission (Figure 1B) and our previously reported finding that YKL-40 levels decrease with immunotherapy (13). When we increased the sample size by combining cohort A and B, we could confirm that the levels of YKL-40 were increased in SPMS as well (Figure 1D). This finding challenges the prevailing view that inflammation is unimportant to the pathogenesis of SPMS, but is very much in line with the recently published study from Komori et al, who reported that markers of B and T cell activation were present in the CSF from RRMS patients and SPMS patients to a similar degree (24).

To investigate YKL-40 in relation to tissue damage a series of correlation analyses were performed. To remove the effect of acute inflammation, we excluded subjects with $\mathrm{Gd}+$ lesions from the analysis. It is well known that T1 lesions may evolve into black holes, which probably is the MRI correlate of a chronic active lesion (23). We found that the T1 lesion load correlated with YKL-40, suggesting that this biomarker may reflect on-going low grade inflammation in such areas. In contrast, classical inflammation driven demyelination is largely absent in the NAWM where instead prominent axonal degeneration is present in SPMS patients (25). Several methods have been used to estimate the integrity of NAWM. In this study, we measured the SI of NAWM in T2 weighted images and normalized the SI against two external references containing either oil or water. A high normalized SI represents increased water content in the NAWM, reflecting tissue damage not visible to the naked eye. We found an inverse relation between the SIs and the levels of YKL-40. Thus our data suggest a fundamental 
difference to the origin of tissue damage in T1 lesions vis-a-vis NAWM. This is consistent with a dual paradigm where both inflammation and degeneration are important to the development of tissue damage and disability in SPMS.

We also investigated how YKL-40 related to other biomarkers of astrocyte and microglia activation. GFAp is the principal intermediate filament in mature CNS astrocytes. GFAp is thought to be important in modulating astrocyte motility and shape by providing structural stability to astrocytic processes. Following tissue injury, astrocytes become reactive and respond with rapid synthesis of GFAp (26). GFAp is an established biomarker of astrogliosis and moderately elevated levels of GFAp have previously been demonstrated in patients with progressive MS (27-30). CD14 is abundantly found on macrophages and monocytes, but also exists in a soluble form (sCD14), which either appears after shedding of membrane bound CD14 or is directly secreted from intracellular vesicles (31). Increased levels of sCD14 in serum have been demonstrated in stable RRMS and SPMS patients $(32,33)$, whereas we recently reported that the levels of sCD14 in CSF from MS patients were similar to healthy controls (13).

In difference to YKL-40, GFAp and sCD14 did not correlate with the number of Gd+ lesions, suggesting that the levels of these biomarkers are independent of acute inflammation. Since YKL-40 is believed to be dependent on both astrocyte and microglia activation, we expected to find a correlation between the levels of YKL-40 and GFAp as well as sCD14. Again, to remove the effect of acute inflammation, we excluded subjects with Gd+ lesions from the analysis. YKL-40, GFAp and sCD14 were all correlated with each other to a similar degree (Table 3). GFAp correlated well with measures of atrophy and to a lesser extent with T1/T2 lesions, but not convincingly with SIs of NAWM. Most likely this is reflecting lesion dependent astrogliosis in the CNS. Somewhat unexpectedly, no significant correlation was found between any MRI parameter and sCD14. Since the levels of sCD14 were also similar between MS patients and controls, the value of sCD14 as a biomarker in MS is questionable at best. A possible explanation to the negative findings is that SCD14 is predominantly produced in the periphery with more than tenfold higher concentration in serum in comparison to CSF $(13,34)$ and the levels of sCD14 in CSF might be influenced by passive transfer over the blood-brain barrier.

Overall, our results are in concordance with previous studies. Several previous investigators have reported YKL40 to be increased in the CSF from MS or CIS patients (9-15). Two studies reported that YKL-40 correlated with the number of $\mathrm{Gd}+$ lesions to a similar degree as in the present study $(10,11)$, while another failed to detect a correlation, most likely due to the low number of enhancing lesions in the cohort. YKL-40 in SPMS has been less 
studied. In one study YKL-40 levels in SPMS were similar to healthy controls (11), which is seemingly at odds with the findings from the present study. The reason for this discrepancy is unclear, but may reflect underlying differences in the composition of the studied cohorts.

\section{Conclusion}

Several biomarkers are available to monitor disease activity in RRMS, but biomarkers of SPMS are few and far between. We have demonstrated that the YKL-40 level is elevated in CSF from SPMS patients, that YKL-40 correlates with the number of T1 lesions and is inversely correlated with the SI of NAWM. This suggests that the CSF level of YKL-40 in SPMS is reflecting the number of chronic active lesions. Such lesions probably contribute significantly to the development of disability in SPMS patients and thus YKL-40 should be a good biomarker to monitor disease activity in SPMS.

\section{Acknowledgements}

The authors would like to thank Monica Christiansson, Sara Hullberg, Åsa Källén, Dzemila Secic, och Jonas Söderblom, who performed the ELISAs. In addition Dr Lisa Christiansson, Dr Hanna Karlsson, Dr Lina Liljenfeldt and Emma Svensson, who assisted with preparation of samples. Maria Bertilsson assisted with statistical analyses. 


\section{References}

1. Lublin F, Reingold S. Defining the clinical course of multiple sclerosis: results of an international survey. Neurology. 1996;46(4):907-11.

2. Sospedra M, Martin R. Immunology of multiple sclerosis. Annual Review of Immunology. 2005;23:683747.

3. Filippi M, Rossi P, Campi A, Colombo B, Pereira C, Comi G. Serial contrast-enhanced MR in patients with multiple sclerosis and varying levels of disability. American Journal of Neuroradiology. 1997;18(8):1549-56.

4. Koziol J, Wagner S, Adams H. Assessing information in T2- weighted MRI scans from secondary progressive MS patients. Neurology. 1998;51:228-33.

5. Confavreux C, Vukusic S, Adeleine P. Early clinical predictors and progression of irreversible disability in multiple sclerosis: an amnesic process. Brain. 2003;126(Pt 4):770-82.

6. Rovaris M, Confavreux C, Furlan R, Kappos L, Comi G, Filippi M. Secondary progressive multiple sclerosis: current knowledge and future challenges. The Lancet Neurology. 2006;5(4):343-54.

7. Prineas JW, Kwon EE, Cho ES, Sharer LR, Barnett MH, Oleszak EL, et al. Immunopathology of secondaryprogressive multiple sclerosis. Ann Neurol. 2001;50(5):646-57.

8. Frischer JM, Bramow S, Dal-Bianco A, Lucchinetti CF, Rauschka H, Schmidbauer M, et al. The relation between inflammation and neurodegeneration in multiple sclerosis brains. Brain. 2009;132(Pt 5):1175-89. 9. Bonneh-Barkay D, Wang G, Starkey A, Hamilton RL, Wiley CA. In vivo CHI3L1 (YKL-40) expression in astrocytes in acute and chronic neurological diseases. J Neuroinflammation. 2010;7:34.

10. Canto E, Tintore M, Villar LM, Costa C, Nurtdinov R, Alvarez-Cermeno JC, et al. Chitinase 3-like 1: prognostic biomarker in clinically isolated syndromes. Brain. 2015;138(Pt 4):918-31.

11. Correale J, Fiol M. Chitinase effects on immune cell response in neuromyelitis optica and multiple sclerosis. Mult Scler. 2011;17(5):521-31.

12. Modvig S, Degn M, Horwitz H, Cramer SP, Larsson HB, Wanscher B, et al. Relationship between Cerebrospinal Fluid Biomarkers for Inflammation, Demyelination and Neurodegeneration in Acute Optic Neuritis. PLoS One. 2013;8(10):e77163.

13. Malmestrom C, Axelsson M, Lycke J, Zetterberg H, Blennow K, Olsson B. CSF levels of YKL-40 are increased in MS and replaces with immunosuppressive treatment. J Neuroimmunol. 2014;269(1-2):87-9. 14. Martinez MA, Olsson B, Bau L, Matas E, Calvo AC, Andreasson U, et al. Glial and neuronal markers in cerebrospinal fluid predict progression in multiple sclerosis. Mult Scler. 2015;21(5):550-61. 
15. Modvig S, Degn M, Roed H, Sorensen T, Larsson H, Langkilde A, et al. Cerebrospinal fluid levels of chitinase 3-like 1 and neurofilament light chain predict multiple sclerosis development and disability after optic neuritis. Mult Scler. 2015.

16. Polman CH, Reingold SC, Banwell B, Clanet M, Cohen JA, Filippi M, et al. Diagnostic criteria for multiple sclerosis: 2010 revisions to the McDonald criteria. Annals of Neurology. 2011;69(2):292-302.

17. Kurtzke JF. Rating neurologic impairment in multiple sclerosis: an expanded disability status scale (EDSS). Neurology. 1983;33(11):1444-52.

18. Rosengren L, Wikkelso C, Hagberg L. A sensitive ELISA for glial fibrillary acidic protein: application in CSF of adults. J Neurosci Methods. 1994;51:197-204.

19. Salonen O, Autti T, Raininko R, Ylikoski A, Erkinjuntti T. MRI of the brain in neurologically healthy middle-aged and elderly individuals. Neuroradiology. 1997;39(8):537-45.

20. Burman J, Svensson E, Fransson M, Loskog AS, Zetterberg H, Raininko R, et al. The cerebrospinal fluid cytokine signature of multiple sclerosis: A homogenous response that does not conform to the Th1/Th2/Th17 convention. J Neuroimmunol. 2014;277(1-2):153-9.

21. Bonneh-Barkay D, Bissel SJ, Wang G, Fish KN, Nicholl GC, Darko SW, et al. YKL-40, a marker of simian immunodeficiency virus encephalitis, modulates the biological activity of basic fibroblast growth factor. Am J Pathol. 2008;173(1):130-43.

22. Katz D, Taubenberger JK, Cannella B, McFarlin DE, Raine CS, McFarland HF. Correlation between magnetic resonance imaging findings and lesion development in chronic, active multiple sclerosis. Ann Neurol. $1993 ; 34(5): 661-9$

23. Giannetti P, Politis M, Su P, Turkheimer F, Malik O, Keihaninejad S, et al. Microglia activation in multiple sclerosis black holes predicts outcome in progressive patients: an in vivo [(11)C](R)-PK11195-PET pilot study. Neurobiology of disease. 2014;65:203-10.

24. Komori M, Blake A, Greenwood M, Lin YC, Kosa P, Ghazali D, et al. CSF markers reveal intrathecal inflammation in progressive multiple sclerosis. Ann Neurol. 2015.

25. Kutzelnigg A, Lucchinetti CF, Stadelmann C, Bruck W, Rauschka H, Bergmann M, et al. Cortical demyelination and diffuse white matter injury in multiple sclerosis. Brain. 2005;128(Pt 11):2705-12.

26. Eng L, Ghirnikar R, Lee Y. Glial fibrillary acidic protein: GFAP-thirty-one years (1969-2000). Neurochem Res. 2000;25(9-10):1439-51.

27. Rosengren L, Lycke J, Andersen O. Glial fibrillary acidic protein in CSF of multiple sclerosis patients relation to neurological deficit. J Neurol Sci. 1995;133(1-2):61-5. 
28. Malmeström C, Haghighi S, Rosengren L, Andersen O, Lycke J. Neurofilament light protein and glial fibrillary acidic protein as biological markers in MS. Neurology. 2003;61(12):1720-5.

29. Norgren N, Sundström P, Svenningsson A, Rosengren L, Stigbrand T, Gunnarsson M. Neurofilament and glial fibrillary acidic protein in multiple sclerosis. Neurology. 2004;63(9):1586-90.

30. Burman J, Zetterberg H, Fransson M, Loskog AS, Raininko R, Fagius J. Assessing tissue damage in multiple sclerosis: a biomarker approach. Acta Neurol Scand. 2014.

31. Kirkland TN, Viriyakosol S. Structure-function analysis of soluble and membrane-bound CD14. Progress in clinical and biological research. 1998;397:79-87.

32. Lutterotti A, Kuenz B, Gredler V, Khalil M, Ehling R, Gneiss C, et al. Increased serum levels of soluble CD14 indicate stable multiple sclerosis. J Neuroimmunol. 2006;181(1-2):145-9.

33. Brettschneider J, Ecker D, Bitsch A, Bahner D, Bogumil T, Dressel A, et al. The macrophage activity marker sCD14 is increased in patients with multiple sclerosis and upregulated by interferon beta-1b. J Neuroimmunol. 2002;133(1-2):193-7.

34. Vrethem M, Kvarnstrom M, Stenstam J, Cassel P, Gustafsson M, Landtblom AM, et al. Cytokine mapping in cerebrospinal fluid and blood in multiple sclerosis patients without oligoclonal bands. Mult Scler.

2012;18(5):669-73. 


\section{Tables}

Table 1. Clinical and demographical data of Cohort A.

\begin{tabular}{lcccc}
\hline & Controls & Gd- RRMS & Gd+ RRMS & SPMS \\
\hline $\mathbf{n}$ & 30 & 18 & 24 & 20 \\
Female/Male & $8 / 22$ & $12 / 6$ & $16 / 8$ & $11 / 9$ \\
Age (IQR) & $40(36-52)$ & $39(34-45)$ & $38(25-44)$ & $59(54-66)$ \\
EDSS (IQR) & $\mathrm{n} / \mathrm{a}$ & $2.0(1.5-2.0)$ & $2.0(1.0-2.9)$ & $5.8(3.6-6.0)$ \\
Disease duration (IQR) & $\mathrm{n} / \mathrm{a}$ & $9.6(7.5-20)$ & $4.7(0.20-7.0)$ & $23(14-29)$ \\
On treatment & $\mathrm{n} / \mathrm{a}$ & $10 / 18$ & $10 / 24$ & $2 / 20$ \\
\hline
\end{tabular}

Gd-, not enhancing gadolinium; Gd+, gadolinium enhancing; RRMS, relapsing-remitting multiple sclerosis; SPMS, secondary progressive multiple sclerosis; EDSS, expanded disability status scale. Age (years), EDSS and disease duration (years) are described as medians. 
Table 2. Correlations between biomarkers of astrocyte and microglia activation and MRI parameters.

\begin{tabular}{l|c|cc|cc|ccc|}
\hline \multicolumn{1}{|c}{ GFAP } & \multicolumn{2}{c}{ YKL-40 } & \multicolumn{2}{c}{ sCD14 } \\
\hline Gd+ & $\mathrm{n}$ & $\mathrm{r}$ & $\mathrm{p}$-value & $\mathrm{R}$ & $\mathrm{p}$-value & $\mathrm{r}$ & $\mathrm{p}$-value \\
\hline \hline T1 & 62 & -0.18 & 0.15 & $\mathbf{0 . 3 3}$ & 0.0097 & -0.080 & 0.54 \\
\hline T2 & 35 & $\mathbf{0 . 5 2}$ & 0.0013 & $\mathbf{0 . 4 4}$ & 0.0071 & 0.18 & 0.18 \\
Atrophy third ventricle & 35 & $\mathbf{0 . 5 2}$ & 0.012 & $\mathbf{0 . 3 5}$ & 0.039 & 0.090 & 0.61 \\
Atrophy lateral ventricle & 35 & $\mathbf{0 . 6 6}$ & $<0.0001$ & 0.27 & 0.12 & 0.0007 & 1.0 \\
Atrophy cerebral sulci & 35 & $\mathbf{0 . 5 8}$ & 0.0004 & 0.28 & 0.11 & 0.13 & 0.47 \\
NAWM cerebrum - oil & 35 & $\mathbf{0 . 7 0}$ & $<0.0001$ & $\mathbf{0 . 3 7}$ & 0.030 & 0.051 & 0.77 \\
NAWM cerebrum - water & 29 & 0.28 & 0.14 & -0.21 & 0.27 & -0.20 & 0.29 \\
NAWM pons and cerebellum - oil & 33 & 0.074 & 0.68 & $\mathbf{- 0 . 4 5}$ & 0.0094 & -0.34 & 0.051 \\
NAWM pons and cerebellum - water & 30 & -0.0022 & 0.99 & $\mathbf{- 0 . 4 1}$ & 0.027 & -0.35 & 0.065 \\
\hline
\end{tabular}

MRI, magnetic resonance imaging; GFAp glial fibrillary acidic protein; Gd+, gadolinium enhancing; NAWM, normal appearing white matter; Significant correlations in bold face. Patients with Gd+ lesions were excluded from the correlation analysis of the parameters below the double line. 
Table 3. Correlations between YKL-40, GFAp and sCD14.

\begin{tabular}{lcccccc}
\hline & \multicolumn{2}{c}{ GFAp } & \multicolumn{2}{c}{ YKL-40 } & \multicolumn{2}{c}{ sCD14 } \\
\hline GFAp & Spearman $r$ & p-value & Spearman $r$ & $p$-value & Spearman $r$ & $p$-value \\
YKL-40 & & & 0.43 & 0.00053 & 0.48 & 0.00012 \\
sCD14 & 0.43 & 0.00053 & & & 0.60 & $<0.0001$ \\
\hline
\end{tabular}

Patients with relapses and gadolinium enhancing lesions were excluded from the analysis. 


\section{Figures}

Figure 1. YKL-40.

A

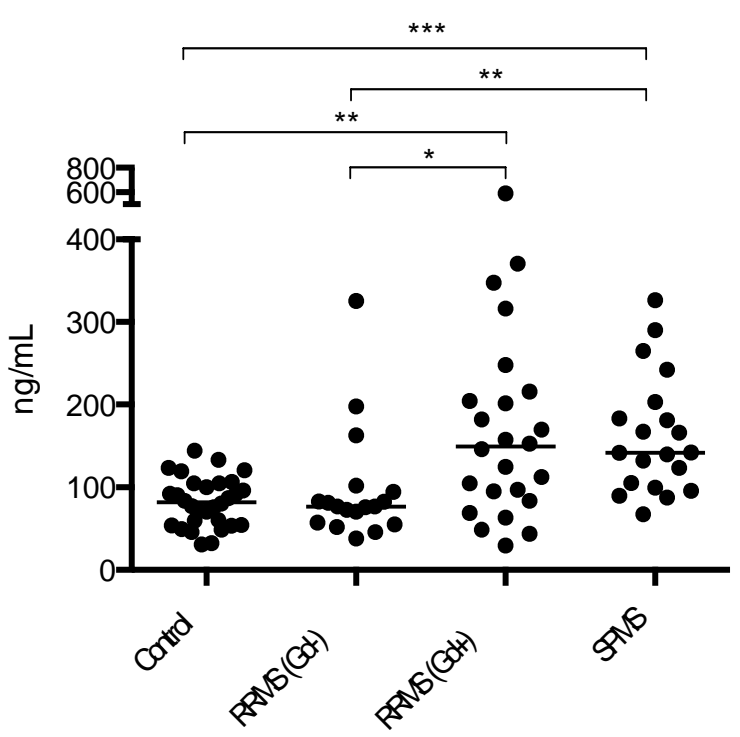

C

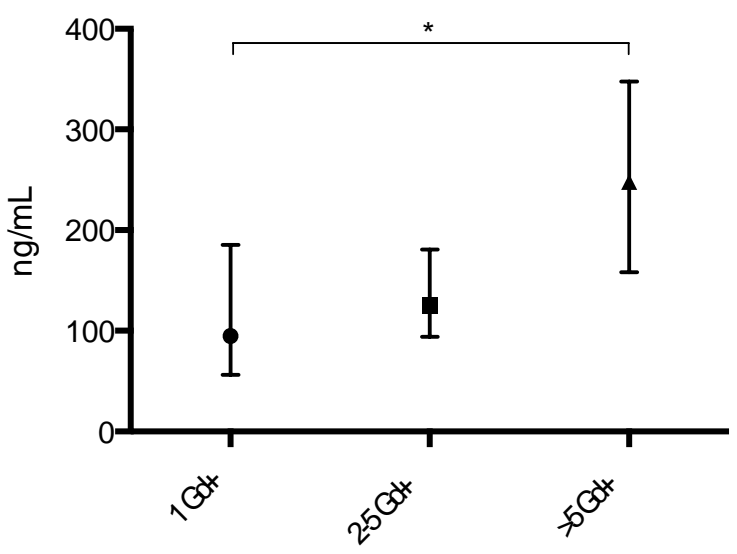

B
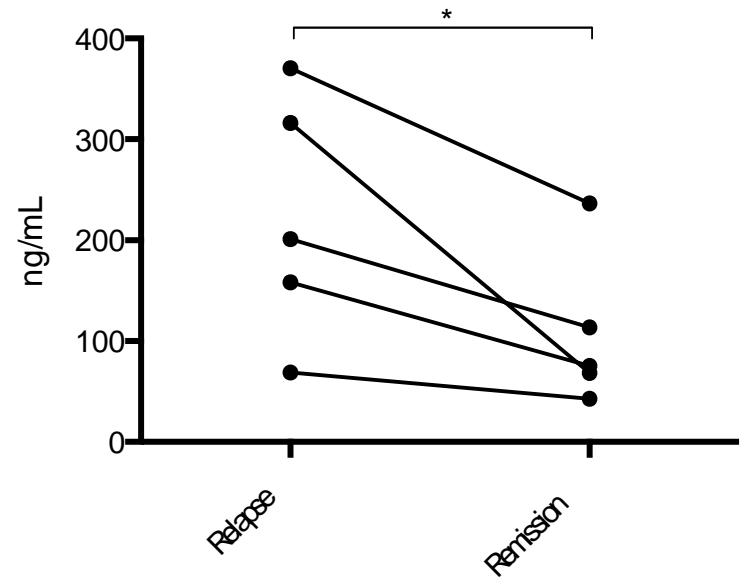

$\mathbf{D}$

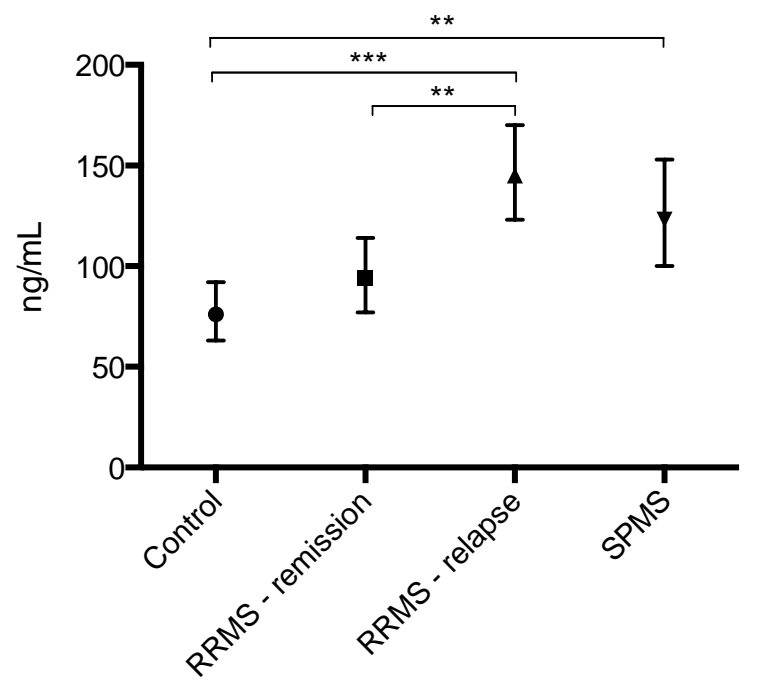

Gd-, not enhancing gadolinium; Gd+, gadolinium enhancing; RRMS, relapsing-remitting multiple sclerosis; SPMS, secondary progressive multiple sclerosis. A) Unadjusted levels of YKL-40 in healthy controls and patients in different stages of MS from cohort A. Each dot represents one individual. B) YKL-40 levels in five RRMS patients sampled in relapse and remission. C) Levels of YKL-40 in RRMS patients with Gd+ lesions (median with IQR). D) Age adjusted analysis of differences in YKL-40 levels in patients from the combined cohorts (geometric mean with $95 \% \mathrm{CI}$ ). 